\title{
Percepções e atitudes de primigestas em relação à atenção em saúde bucal materno-infantil: um estudo qualitativo
}

\author{
Igor Henrique Teixeira FUMAGALLI'; Luana Pinho de Mesquita LAGO²0; \\ Soraya Fernandes MESTRINER®i ; Alexandre Fávero BULGARELLI4; \\ Wilson MESTRINER JÚNIOR ${ }^{5}$
}

1 - Mestrando do programa de Pós-graduação em Saúde Pública da Escola de Enfermagem de Ribeirão Preto da Universidade de São Paulo (USP); 2 - Professora da Faculdade de Odontologia de Ribeirão Preto, Departamento de Estomatologia, Saúde Coletiva e Odontologia Legal da Universidade de São Paulo (USP); 3 - Professora Doutora da Faculdade de Odontologia de Ribeirão Preto, Departamento de Estomatologia, Saúde Coletiva e Odontologia Legal da Universidade de São Paulo (USP); 4 - Professor Associa do da Faculdade de Odontologia da Universidade Federal do Rio Grande do Sul (UFRGS), Departamento de Odontologia Social e Preventiva; 5 - Professor Titular da Faculdade de Odontologia de Ribeirão Preto, Departamento de Estomatologia, Saúde Coletiva e Odontologia Legal da Universidade de São Paulo (USP).

\section{Resumo}

Objetivo: Identificar crenças de mulheres primigestas que influenciam na procura por assistência odontológica e adoção de cuidados relativos à sua saúde bucal e dos filhos. Material e método: Estudo descritivo com metodologia qualitativa. Foram realizadas entrevistas com roteiro semi-estruturado, que foram audiogravadas e posteriormente transcritas, com 20 primigestas cadastradas em seis unidades de saúde da família de um município do Estado de São Paulo. Os dados foram categorizados pela técnica da Análise de Conteúdo e analisados com suporte do Modelo de Crenças em Saúde. Resultados: Foram identificadas três categorias: Percepções dos riscos e benefícios na primeira gestação, Gravidade percebida e barreiras na primeira gestação, Superação de barreiras e adoção de cuidados em saúde bucal. As gestantes acreditavam na suscetibilidade à transmissão de doenças durante a gestação, na possibilidade dos procedimentos odontológicos e certos hábitos apresentarem riscos à sua saúde e influenciarem no desenvolvimento do bebê. As principais barreiras apontadas foram o medo dos procedimentos odontológicos e a recusa ao atendimento à gestante, enquanto a família, as redes sociais e grupos de promoção de saúde foram os principais meios informativos. Conclusões: A aproximação às percepções, crenças e atitudes de primigestas pode ampliar os conhecimentos sobre os cuidados em saúde, revelar mitos, hábitos e aprendizagens neste período, contribuir no planejamento de ações pautadas no diálogo e fortalecimento de vínculos entre profissionais da equipe e gestantes, e na promoção de cuidados em saúde bucal durante o pré-natal.

Copyright @ 2021 Revista

PALAVRAS-CHAVE: Saúde bucal; Gestantes; Pré-natal; Percepção; Atitude. 


\section{Introdução}

As crenças sobre o período gestacional têm determinado mudanças na percepção e atitudes de mulheres, de forma que julgamentos a respeito dos cuidados em saúde bucal e o grau de importância que as gestantes dão a estes podem refletir em seu distanciamento dos serviços de saúde ${ }^{1}$. Nesse período acontecem diversas alterações fisiológicas e psicológicas na mulher, especialmente em primigestas ${ }^{2}$, e, este constitui-se como momento oportuno a mudanças de hábitos e importante para busca por informações referente aos cuidados relacionados com sua saúde e, principalmente, com a do bebê $\hat{e}^{3}$.

Estudos ${ }^{4,5}$ apontam a importância da abordagem de crenças e atitudes nesse período, relacionando-os ao planejamento de ações em saúde bucal e educação em saúde que possam atender às necessidades referidas pelas gestantes e promover a sensibilização ao cuidado em saúde bucal. Assim, nesses estudos, as gestantes expressaram sua busca por atendimento odontológico, a adoção de cuidados com a saúde bucal e o quanto creem em sua importância neste momento de sua vida.

Nesse sentido, o cuidado pré-natal é uma importante estratégia que envolve ações de promoção de saúde, diagnóstico e prevenção de doenças, e busca estabelecer uma comunicação efetiva entre equipe de saúde e gestantes sobre aspectos socioculturais, elementos fundamentais para a saúde materna e do recém-nascido $^{6}$. O cuidado pré-natal está previsto, no âmbito do Sistema Único de Saúde (SUS), por meio da política de atenção materno-infantil, ou Rede Cegonha, que tem como objetivo reduzir a taxa de morbimortalidade materno-infantil, ao implementar um modelo de atenção à saúde da mulher e da criança centrado em suas necessidades ${ }^{7}$.

Para atender às demandas, os serviços de saúde ofertam consultas de pré-natal odontológico, ação importante para que as gestantes possam esclarecer suas dúvidas sobre o tratamento odontológico, participar na tomada de decisão quanto aos 
procedimentos a serem realizados e, principalmente, ampliar seus conhecimentos sobre práticas de prevenção, promoção e recuperação da saúde ${ }^{8}$. Dessa forma, pode-se favorecer o cuidado integral e a clínica ampliada ${ }^{9,10}$.

Por outro lado, crenças e mitos de que o tratamento possa ser prejudicial ao bebê, a expectativa da dor e a insegurança podem resultar em baixa adesão ao pré-natal odontológico, associado aos complicadores do acesso, aspectos socioeconômicos, culturais e educacionais ${ }^{11}$.

Deste modo o presente estudo é norteado pelas questões de pesquisa: como mulheres primigestas percebem suas atitudes em relação à sua saúde bucal? Estas percepções podem nortear atitudes em relação aos cuidados à sua saúde bucal e a de seu futuro filho? É neste contexto que a presente pesquisa tem por objetivo identificar as crenças, percepções e atitudes de mulheres primigestas que influenciam na procura por atendimento odontológico e na adoção de cuidados em saúde bucal.

\section{Método}

Estudo descritivo de abordagem metodológica qualitativa, realizado em um município do Estado de São Paulo, Brasil. Este município tinha uma população de aproximadamente 703.293 habitantes em $2019^{12}$, e sua rede de prestação de serviços do SUS encontra-se distribuída por cinco Distritos de Saúde. O estudo foi realizado com gestantes cadastradas em seis Unidades de Saúde da Família (USF) do Distrito Oeste deste município, território com população estritamente urbana.

Estas USFs seguem o modelo da Estratégia Saúde da Família (ESF), desenvolvendo as práticas do cuidado na atenção básica, com equipes multiprofissionais. Cabe ressaltar que desenvolvem atividades acadêmico-assistenciais vinculadas à Universidade que desenvolveu a presente pesquisa. Embora não possuam equipes de saúde bucal cadastradas pelo modelo tradicional do 
Ministério da Saúde, cirurgiões-dentistas e residentes odontólogos compõem as equipes com compromisso de coordenação do cuidado em saúde bucal.

Optou-se por uma amostra intencional buscando todos os casos típicos (gestantes primigestas) cadastradas nas 6 USFs. Com esse pressuposto, todas as gestantes provenientes do programa de pré-natal foram selecionadas a participar do estudo, em um total de 25. Destas, 20 aceitaram participar após a leitura e assinatura do Termo de Consentimento Livre e Esclarecido (TCLE), e 5 não foram encontradas após três tentativas.

As entrevistas foram realizadas no período de março de 2015 a agosto de 2016, por um único entrevistador, estudante de graduação em odontologia, externo ao convívio e sem vínculo com as entrevistadas. A duração média das entrevistas foi de $30 \mathrm{mi}-$ nutos, realizada no domicílio das gestantes ou na USF, com a presença somente das entrevistadas e entrevistador, guardada a privacidade necessária.

Utilizou-se um questionário de caracterização do perfil da população que incluiu idade, estado civil, escolaridade, naturalidade, período gestacional, unidade de saúde de referência para o acompanhamento do pré-natal e informações sobre uso de serviços odontológicos. Foi utilizado um roteiro semiestruturado com abordagem de questões referentes à: obtenção de informações a respeito da saúde bucal durante o pré-natal, acompanhamento pré-natal e participação de membros da equipe multiprofissional no cuidado pré-natal e pré-natal odontológico, questões relacionadas à auto percepção de saúde bucal, hábitos de saúde bucal e alimentares, cuidados com a saúde bucal do bebê e participação de atividades na unidade de saúde da família.

As entrevistas foram audiogravadas e transcritas e os materiais textuais produzidos com as transcrições das entrevistas foram analisados em ordem aleatória, e categorizados por 
meio da técnica de Análise de Conteúdo $(\mathrm{AC})^{13}$. Para tanto, foram realizadas leituras flutuantes, elaboração de hipóteses temáticas com construção de categorias iniciais, recategorização e inferências com apoio no Referencial Teórico do Modelo de Crenças em Saúde (MCS) ${ }^{10-12}$ e construção final dos temas a serem discutidos?.

O MCS é composto por seis dimensões que se inter-relacionam ${ }^{14-16}$ : Percepção de suscetibilidade, severidade, benefícios e barreiras; Estímulo para ação; Autoeficácia. As dimensões foram representadas por palavras, frases, orações e temas categorizados.

Esta pesquisa foi aprovada pelo Comitê de Ética e Pesquisa da Faculdade de Odontologia de Ribeirão Preto da Universidade de São Paulo sob o protocolo nº CAAE: 42788315.5.0000.5419.

\section{Resultados}

As gestantes entrevistadas apresentam um perfil de mulheres jovens, com faixa etária entre 15 e 35 anos e escolaridade variável: ensino médio completo (35\%), ensino médio incompleto $(35 \%)$, ensino fundamental completo (10\%), ensino fundamental incompleto (20\%) e nenhuma com ensino superior. De acordo com o estado civil, $30 \%$ eram solteiras, $15 \%$ casadas e $55 \%$ com união estável. As mesmas apresentaram naturalidades diversas: dez delas nasceram no município do estudo (SP) e as demais em outras cidades de São Paulo (SP), Minas Gerais (MG), Distrito Federal (DF), Alagoas (AL), Bahia (BA) e Rio Grande do Sul (RS).

A obtenção de informações a respeito da saúde bucal durante o pré-natal, evidenciou que seis delas tiveram contato com o cirurgião-dentista, ou equipe da USF durante este período, ou tiveram suporte das redes sociais; quatro consultaram com o dentista, e as demais não tiveram a oportunidade da consulta, seja por ausência do profissional em sua unidade de saúde ou do encaminhamento de um profissional da equipe multiprofissional para um serviço de assistência odontológica. 
O perfil das participantes caracteriza-se por serem jovens com razoável grau de escolaridade, provenientes de diferentes regiões do país que, na sua maioria, não tiveram acesso a informações relacionadas à saúde bucal durante o pré-natal na USF, e residentes em um território que abriga uma população em situação de vulnerabilidade econômica, social e mental ${ }^{17}$.

A seguir, os resultados são apresentados na forma textual em três categorias temáticas intituladas como: Percepções dos riscos e benefícios na primeira gestação; Gravidade percebida e barreiras na primeira gestação; Superação de barreiras e a adoção de cuidados em saúde bucal.

\section{Percepções dos riscos e benefícios na primeira gestação}

A percepção da presença de riscos durante a primeira gestação está relacionada à susceptibilidade, ou, ao caráter subjetivo do risco pessoal de contrair uma condição ${ }^{14}$. Utilizou-se este conceito relacionando-o ao momento vivido na gravidez de mulheres em primeira gestação e os cuidados de saúde bucal. As gestantes se percebem suscetíveis quando creem na relação sistêmica entre mãe e bebê e que seus hábitos, e sua condição de saúde, podem afetar o bebê: "qualquer coisa que eu pegar vai transmitir pro bebê" (ENTREVISTA 5).

É importante considerar que estas crenças podem ser também dos profissionais de saúde em relação à saúde bucal:

[...] (a enfermeira) falou pra mim que (quando se trata de) infecção de dente, tem um estudo ... ela (a enfermeira) [...] falou que foi comprovado que vai direto pro bebê (ENTREVISTA 12).

Outra dimensão das crenças diz respeito à relação nutricional mãe-bebê durante a gestação: "geralmente o cálcio vai pro nenê né? que me falaram. Então vai ficando bem mais fraco os dentes da gente" (ENTREVISTA 4). 
A entrevistada 4 acredita que seu dente pode enfraquecer, perder as propriedades, à medida que "passa” o cálcio para o feto, além disso, as gestantes creem que é possível haver a transmissão de microorganismos e infecções ou outros acometimentos da boca da mãe para o feto.

Ainda quanto à crença na relação causa e efeito, uma das gestantes fazia uso de balas de mascar para aliviar um desconforto abdominal, e relacionou seu hábito ao desenvolvimento da Diabetes Mellitus no feto: "Ah porque estraga os dentes e já deve fazer mal pro bebê e ainda desenvolver uma diabetes” (ENTREVISTA 7).

Além das condições de saúde bucal e hábitos, as participantes entendem que os procedimentos odontológicos podem trazer, em sua maioria, riscos à sua saúde e ao desenvolvimento do bebê. Sobre a possibilidade de frequentar o dentista durante a gestação, algumas gestantes a associam diretamente ao tratamento odontológico e acreditam que não seja recomendável realizar alguns procedimentos, como a exposição durante um exame radiográfico, e ao mesmo tempo, reconhecem seu desconhecimento em relação a outras condutas: "Eu sei que raio $\mathrm{X}$ não pode fazer. Mas [...] a respeito do tratamento do dente mesmo eu não sei” (ENTREVISTA 12).

Além dos riscos, as primigestas percebem os benefícios advindos da adoção de cuidados durante a gestação. De acordo com o MCS, neste período determinadas ações são mais efetivas ou tem consequências positivas, uma vez que a adoção de novos hábitos é percebida como viável e eficaz ${ }^{14}$.

Um exemplo é a percepção de que as doenças bucais podem ser prevenidas durante a gestação: "Então é você já tem que cuidar no normal, grávida você tem que cuidar o dobro" (ENTREVISTA 2). Quando questionadas sobre os futuros cuidados com a saúde bucal dos filhos, as gestantes percebem o quanto suas atitudes e a participação de um cirurgião-dentista seriam importantes durante este período: 
[...] com a escovinha né, bem levinho né para já desenvolver desde criança para não ter o hábito de não escovar né... tem que ir mostrando pra criança desde pequeno né? Porque depois tem muita criança aí que já vem a cárie né? Desde pequeno (ENTREVISTA 7).

[...] eu acho que para toda grávida deveria de ter (acompanhamento do dentista). Eu não digo participar em todas as consultas (de pré-natal), mas pelo menos uma sim outra não eu acredito que seria bom (ENTREVISTA 2).

Identifica-se que, de modo geral, as gestantes valorizam a participação do profissional cirurgião-dentista durante o pré-natal, estão sensibilizadas quanto aos benefícios dos hábitos de higiene, principalmente a escovação, para a diminuição dos riscos às doenças bucais como a cárie.

\section{Gravidade percebida e barreiras na primeira gestação}

Este tema refere-se à percepção da severidade, ou seja, sentimentos relativos à gravidade de contrair uma doença (ou de deixá-la sem tratamento $)^{14}$. No MCS, estes sentimentos podem variar de pessoa para pessoa e inclui avaliação de consequências clínicas como a morte, incapacidade e dor. E também possíveis consequências sociais como efeitos das condições sobre o trabalho, a vida familiar e as relações sociais.

As gestantes entrevistadas percebem que, de certa forma, algumas atitudes mudaram no seu cotidiano com a gravidez, como hábitos alimentares, lazer, estudos, hábitos ilícitos e restrição a medicamentos:

"Porque quando dá dor no dente não pode beber remédio né? Por causa do nenê" (ENTREVISTA 6);

"Deixei de ir para balada, deixei de beber, deixei de fumar" (ENTREVISTA 8);

"Eu deixei de fazer, tipo, eu fumava, eu não vou mentir para você não, eu fumava” (ENTREVISTA 10);

"[...] estudo eu parei né, o terceiro ano, e eu estava fazendo curso de Administração também e eu parei” (ENTREVISTA 11). 
Em relação a mudanças na boca, relataram o ressecamento da boca no período noturno: "Eu acordo com a boca muito rachada. Acho que é muita sede que eu fico, eu tomo água, mas depois eu acordo com a boca machucada” (ENTREVISTA 10).

Outro aspecto importante apontado pelas gestantes são as barreiras baseadas em suas crenças, sentimentos e em fragilidades estruturais, que podem atuar como impedimentos ao acesso. Algumas delas se referem à indisponibilidade de insumos e recursos humanos, à postura do profissional cirurgião-dentista em não incentivar procedimentos durante a gestação, ou à falta de informação. Uma primigesta relatou um episódio em que o serviço público de saúde havia sido roubado e não havia condições de atendimento e por isso ela optou em utilizar um serviço particular: "porque eu fui ali e tinham roubado uns aparelhos no dia que eu fui e eu peguei e fui no particular" (ENTREVISTA 4).

Em outra situação, o serviço de saúde estava sem disponibilidade de atendimento pela falta de materiais e insumos e novamente a gestante utilizou o serviço odontológico particular e não finalizou o tratamento: "aí depois não pôde... estava sem os materiais pra fazer. Eu fui no particular e fiz um, o outro eu não tratei” (ENTREVISTA 4).

Outra barreira mencionada foi relacionada ao sentimento de que a experiência do tratamento odontológico pode ser perigosa ou dolorosa, explicitada como medo do dentista: "Porque eu morro de medo de dentista” (ENTREVISTA 1). Nesse caso, sentimentos como o medo podem influenciar na busca por cuidados de saúde.

\section{Superação de barreiras e a adoção de cuidados em saúde bucal}

As crenças, as percepções e as atitudes das primigestas podem contribuir na tomada de decisão em relação aos cuidados de saúde bucal. Estas ações podem ser por estímulos de origem "interna, por exemplo, advindo de um sintoma como dor ou 
externa, como influência da família, dos amigos, dos meios de comunicação"16.

No presente estudo, os estímulos para buscar o atendimento odontológico foram a dor e o sentimento de que sua severidade poderia ter consequências para o bebê: "por isso também que eu fui e aguentei, porque antes eu estava com dor e não fui” (ENTREVISTA 4).

Nessa dimensão a oferta de assistência odontológica à população é limitada, há fragilidades na rede de atenção que impedem o acesso aos serviços de saúde bucal. A ausência de referência e/ ou a não orientação de membros da equipe de saúde em relação aos cuidados de saúde bucal contribuem para desestimulá-las à ação, geram dúvidas de como proceder em relação aos cuidados à saúde bucal, levando as gestantes a procurar por orientação e estímulo na família:

Entrevistador: Durante o pré-natal você não foi encaminhada pro Dentista então? Entrevistado: Não (ENTREVISTA 11)

Entrevistador: E ela (enfermeira) também não te orientou como fazer a higienização da boca do nenê? Entrevistado: Não (ENTREVISTA 11)

Quanto à busca por informações sobre a higienização da boca do seu filho, a gestante, diante de seus valores familiares, cita sua mãe: "Acho que com a minha mãe mesmo" (ENTREVISTA 11).

A crença na eficácia dos hábitos saudáveis foi incluída posteriormente ao $\mathrm{MCS}^{16}$ e se relaciona a questões individuais para a superação de barreiras e adoção de cuidados com a saúde, ou seja, no quanto o indivíduo acredita "na sua capacidade de realizar com sucesso e qualidade determinada atividade"16. No contexto desta pesquisa, foram relatadas ações de autocuidado e cuidados com a saúde bucal do bebê com base nas crenças e saberes familiares destas gestantes:

[...] de manhã a hora que eu acordo eu escovo normal né, passo enxaguante bucal e vou trabalhar. Aí a hora que eu como eu escovo passo, porque não tem jeito, porque tem aparelho $\mathrm{e}$ 
tem que passar fio, e toda vez que eu como eu escovo os dentes (ENTREVISTA 2).

[...] quando a gente amamenta a gente tem que estar limpando a linguinha né, do nenê, com paninho, pra tirar o branco da língua (ENTREVISTA 10).

[...] a minha mãe que fala que tem que limpar a boca com a fralda, com o algodão (ENTREVISTA 12).

Por outro lado, foi relatada uma situação em que a gestante utiliza os conhecimentos familiares, e acredita que a ingestão de medicamentos pode ser prejudicial ao bebê e por isso prefere utilizar o medicamento diretamente no dente, desconhecendo a relação sistêmica e os riscos desta prática para o bebê: "às vezes pingava uma Dipirona no dente, colocava um algodãozinho, coisa que foi minha mãe que me ensinou. Mas tomar não. Ficava com a dor." (ENTREVISTA 4).

Essas atitudes em relação à sua saúde foram apreendidas com os familiares, principalmente com a mãe, ou por meio das redes sociais e aplicativos, evidenciando o uso da tecnologia para obtenção e troca de informações com outras gestantes: "Eu participo bastante de grupo de gestante, tanto no (rede social) como no (aplicativo de mensagens)" (ENTREVISTA 7).

\section{Discussão}

Os resultados deste estudo apontam percepções de riscos e benefícios, principalmente quanto à transmissibilidade de doenças e adoção de hábitos que podem influenciar no desenvolvimento do bebê. Por outro lado, o medo dos procedimentos odontológicos e fragilidades de acesso durante a gestação foram percebidos como barreiras. Os conhecimentos obtidos por meio da família, redes sociais e grupos de promoção de saúde foram estímulos para superação de barreiras e adoção de cuidados em saúde bucal.

No contexto vivenciado pelas participantes, a atenção à saúde bucal durante a gestação é muitas vezes negligenciada, 
principalmente devido à vulnerabilidade social. Segundo Martinelli et al..$^{18}(2020)$ baixa escolaridade e renda influenciam as gestantes a utilizar com menor frequência os serviços de saúde e por isso, elas têm menor regularidade nas consultas de pré-natal, fazendo-se necessário o planejamento de buscas ativas pela equipe.

A equipe de saúde investe no fortalecimento de um modelo de cuidado com base na clínica ampliada, com inserção de estudantes de graduação e residentes em cenários de prática na atenção básica, reafirmando achados da literatura que defendem que cuidados em saúde bucal devem ocorrer de forma integrada com as ações da equipe multiprofissional ${ }^{10} \mathrm{e}$ com abordagem ampliada de saúde.

Os cuidados em saúde bucal no período gestacional podem prevenir infecções que poderiam disseminar-se pela corrente sanguínea, importante fator de risco para complicações gestacionais como parto prematuro, recém-nascidos de baixo peso e pré-eclâmpsia ${ }^{19}$. Nos resultados desta pesquisa, as gestantes percebem esses riscos e benefícios, principalmente relacionados à relação sistêmica entre a sua saúde e a saúde do feto, nesse sentido, Zazé et al..$^{20}$ (2014) indicam que o maior risco de transmissão de doenças bucais deve ocorrer no contato oral íntimo da mãe com o filho/bebê, por meio da transmissão vertical e não durante a gravidez.

A literatura aponta que o pré-natal odontológico é de fundamental importância para que a gestante possa se empoderar frente à adoção de novos cuidados e atuar como agente de educação em seu núcleo de convivência ${ }^{21,22}$. A participação de diferentes profissionais da equipe nesse momento, com destaque para a atuação do profissional de saúde bucal, em uma perspectiva dialógica, pode ampliar o conhecimento sobre os cuidados de saúde da família ${ }^{23}$. Essas ações podem interferir em aspectos comportamentais como, por exemplo, na dieta alimentar e composição nutricional logo nos primeiros anos de vida da criança ${ }^{24}$. 
A partir da percepção de susceptibilidade a acometimentos na boca, as gestantes entendem que necessitam de tratamento, porém, acreditam que alguns procedimentos odontológicos, podem trazer riscos à sua saúde e ao desenvolvimento do bebê, por acreditarem que a radiação poderia trazer algum prejuízo ao feto. Porém, pesquisadores ${ }^{25}$ defendem que as tomadas radiográficas, em sua correta indicação e proteção, podem ser realizadas no período gestacional e não devem ser motivo de preocupações para as gestantes. Por outro lado, o profissional cirurgião-dentista por vezes evita realizar o procedimento para reduzir riscos e responsabilizações judiciais caso haja alguma consequência para o bebê, ou até mesmo por mitos pessoais ${ }^{26}$.

Outro aspecto apontado pelas gestantes foi a sensação de boca seca, que apesar de seu caráter subjetivo, pode apresentar-se como risco para alterações e doenças bucais. Durante a gestação, a xerostomia no período noturno é uma possível consequência da diminuição da função das glândulas salivares, com alterações seja na quantidade, ou na qualidade da saliva ${ }^{27}$. No entanto, a literatura aponta que a taxa de fluxo e pH salivar são semelhantes entre gestantes e não gestantes ${ }^{28}$. Assim, é importante considerar a subjetividade presente neste período da vida dessas mulheres e os riscos à saúde bucal.

Nessa pesquisa, algumas primigestas apontaram fragilidades e barreiras de acesso a informações e cuidados de saúde bucal, corroborando os resultados de um estudo com gestantes na atenção básica, que demonstra que a maioria das gestantes não foi atendida pelo cirurgião-dentista no transcorrer da gestação e que há um distanciamento entre elas e os profissionais de saúde bucal ${ }^{29}$.

Em uma perspectiva de adoção de novos hábitos, as gestantes percebem o quanto a gravidez alterou o seu cotidiano, principalmente em relação à hábitos e/ou vícios, e se mostraram preocupadas com as consequências da continuidade destes. De fato, ressalta-se que, se fumantes, as primigestas fazem parte 
do grupo mais adepto ao abandono do hábito de fumar ${ }^{30}$. Para as primigestas deste estudo, a mudança de hábitos geralmente relaciona-se com a preservação da saúde do bebê, por impossibilidade de realizar as atividades devido às condições físicas decorrentes da gravidez, ou pela obtenção de novos conhecimentos neste período.

O pré-natal odontológico, além de colaborar com o nascimento e desenvolvimento seguro da criança ${ }^{23-31}$, pode influenciar as mães no cuidado de si e de seus filhos, apoiando as decisões em relação à realização de procedimentos odontológicos que em seu imaginário poderiam causar algum mal, ou ter consequências como hemorragias, desmaios ou até perda do bebê $\hat{e}^{29}$. Apesar das barreiras estruturais que foram explicitadas pelas gestantes, há incentivo para a inclusão do profissional cirurgião-dentista nos atendimentos de pré-natal nestas unidades.

Muitos profissionais evitam realizar o atendimento odontológico em mulheres grávidas devido à insegurança, mitos e medos apesar de serem os agentes que podem desmitificá-lo ${ }^{1}$. Ainda, outros aspectos que dificultam o cuidado integral são pouco diálogo e problematização sobre a importância do tratamento, além de falta de aprofundamento teórico por parte dos cirurgiões-dentistas ${ }^{31}$. Nesse contexto, as universidades têm papel fundamental na formação de profissionais capacitados e aptos para realizarem as intervenções de saúde em gestantes, preparando-os para o desenvolvimento de práticas educativas ${ }^{1}$ e educação permanente com a equipe.

Na maioria das vezes, é necessário para as gestantes estímulos internos e/ou externos para desencadear o cuidado em saúde, já que as percepções de suscetibilidade e severidade da doença podem criar a intenção para a ação, porém, por si só não garantem sua execução ${ }^{1}$. Robles, Grosseman e $\operatorname{Bosco}^{32}$ (2010) afirmam que a busca pelo dentista para cuidados odontopediátricos ocorre, principalmente, para realização de procedimentos curativos e 
que a jornada de trabalho e as dificuldades financeiras são fatores que dificultam esta procura e os cuidados de saúde.

A superação de barreiras e a adoção de cuidados com a saúde perpassou o suporte familiar e da equipe de saúde, e questões do acesso e do uso das tecnologias em favor da saúde, já que a comunicação se dá cada vez mais por diferentes canais como redes sociais e aplicativos de celular ${ }^{33}$. Contudo, apesar de vivenciarem uma intensa troca de informações via internet, a comunicação entre profissional e gestantes deve ser priorizada durante o pré-natal, evitando a permanência de mitos.

Durante a gestação, o cuidado em saúde bucal implica responsabilidade, compromisso contínuo estendido à família ${ }^{34}$, e de sua rede de apoio social. Assim, os grupos de gestantes tem um papel fundamental ${ }^{35}$, uma vez que são estratégias para construção de vínculo com as equipes e acesso às informações sobre saúde bucal. No entanto, é importante refletir sobre o quanto as gestantes têm voz ativa nesses espaços e se as ações da equipe alcançam suas expectativas e ampliam a clínica e a promoção de saúde ${ }^{36}$.

Ainda, apesar deste estudo abordar especificamente a perspectiva das primigestas sobre o cuidado em saúde bucal, a utilização de entrevistas nesta pesquisa possibilitou exercitar a escuta às gestantes entrevistadas, explorando percepções do processo saúde-doença, do acesso ao cuidado em saúde bucal e dos vínculos construídos ou não com as equipes de saúde, de forma a ampliar a discussão com os profissionais sobre o planejamento e qualificação de ações a essa população.

\section{Conclusões}

A aproximação às crenças, percepções e atitudes na primeira gestação possibilitou ampliar os conhecimentos sobre os cuidados com a saúde bucal neste período, revelar mitos relacionados à transmissão de doenças durante a gestação e a realização de 
procedimentos odontológicos. Para estas gestantes, alguns hábitos poderiam gerar riscos à sua saúde e ao desenvolvimento do bebê e suas atitudes envolveram conhecimentos e aprendizados familiares, que em muitos casos são transmitidos por gerações ou por meio de grupos em redes sociais virtuais.

Por fim, espera-se que este estudo possa instigar os diferentes atores envolvidos no cuidado materno-infantil no SUS, no planejamento de ações pautadas no diálogo e fortalecimento de vínculos entre profissionais da equipe e gestantes, e na promoção de cuidados em saúde bucal durante o pré-natal.

Apesar deste estudo não ter ampliado sua análise para outros determinantes de saúde como a renda familiar, e ter privilegiado a percepção da mãe no cuidado em saúde bucal, considera-se importante incluir aspectos econômicos e culturais, bem como a visão de outros atores como os pais, familiares e profissionais de saúde das equipes de saúde em investigações futuras.

\section{Referências}

1 - Figueira TR, Ferreira EF, Schall VT, Modena CM. O modelo de crenças em saúde e o processo saúde-doença-cuidado bucal por gestantes. ROBRAC. 2013; 22(63): 169-173.

2 - Nunes GS, Leite KNS, Lima TNFA, Paulo APDS, Souza TA, Nascimento BB, et al. Sentimentos vivenciados por primigestas. Rev Enferm UFPE online. 2018; 12(4): 916-22. doi: https://doi. org/10.5205/1981-8963-v12i4a231096p916-922-2018

3 - Bastiani C, Cota ALS, Provenzano MGA, Fracasso MLC, Honório HM, Rios D. Conhecimento das gestantes sobre alterações bucais e tratamento odontológico durante a gravidez. Odontol Clín Cient. 2010; 9(2): 155-160.

4 - Jeihooni AK, Jamshidi H, Kashfi SM, Avand A, Khiyali Z. The Effect of Health Education Program Based on Health Belief Model on Oral Health Behaviors in Pregnant Women of Fasa City, Fars Province, South of Iran. J Int Soc Prev Community Dent. 2017; 7(6): 336-343. doi: 10.4103/jispcd.JISPCD_339_17

5 - Poshtamsary SN, Sigaroudi AE, Farmanbar R, Radafshar G, Roushan ZA. The Effect of Educational Intervention Based on the Health Belief Model on Oral and Dental Health Behavior in Pregnant Women. J Holist Nurs Midwifery. 2020; 30(3): 182-190. 
6 - Organização Mundial da Saúde (OMS). Recomendações da OMS sobre cuidados pré-natais para uma experiência positiva na gravidez. Organização Mundial da Saúde; 2016.

7 - Cassiano ACM, Carlucci EMS, Gomes CF, Bennemann RM. Saúde materno infantil no Brasil: evolução e programas desenvolvidos pelo Ministério da Saúde. RSP. 2014; 65(2): 227-244. doi: https://doi. org/10.21874/rsp.v65i2.581

8 - Saliba TA, Custódio LBM, Saliba NA, Moimaz SAS. Dental prenatal care in pregnancy. Rev Gaúch Odontol. 2019; 67: e20190061. doi: http:// dx.doi.org/10.1590/1981-863720190006120180003

9 - Brasil. Ministério da Saúde. Secretaria de Atenção à Saúde. Departamento de Atenção Básica. Coordenação Nacional de Saúde Bucal. Diretrizes da Política Nacional de Saúde Bucal. Brasília, DF: Ministério da Saúde; 2004.

10 - Rocha NB, Veltrini VC, Bispo CGC, Pielarisi N, Terada RSS, Fujimaki M. Processo de construção coletiva da Clínica Ampliada na Odontologia por meio de uma gestão colegiada. Rev ABENO. 2017; 17(4): 121-132. doi: https://doi.org/10.30979/rev.abeno.v17i4.511

11 - Silva CC, Savian CM, Prevedello BP, Zamberlan C, Dalpian DM, Santos BZ. Acesso e utilização de serviços odontológicos por gestantes: revisão integrativa de literatura. Ciênc Saúde Colet. 2020; 25(3): 827835. doi: http://dx.doi.org/10.1590/1413-81232020253.01192018

12 - Brasil. Ministério da Economia. Instituto Brasileiro de Geografia e Estatística - IBGE. Estimativas da população residente com data de referência $1^{\circ}$ de Julho de 2018. IBGE, 2018.

13 - Bardin L. Análise de conteúdo. São Paulo: Edições 70; 2011. 229 p.

14 - Rosenstock IM. Historical origins of the health belief model. Health education monographs 1974; 2(4):328-345. doi: https:/doi. org/10.1177/109019817400200403

15 - Janz NK, Becker MH. The Health Belief Model: a decade later. Health Education Quarterly. 1984; 11(1): 1-47. doi: https:/doi. org/10.1177/109019818401100101

16 - Rosenstock IM, Strecher VJ, Becker MH. Social learning theory and the health belief model. Health education quarterly. 1988; 15(2): 175183. doi: https://doi.org/10.1177/109019818801500203

17 - Mussolin MG, Mesquita-Lago LP, Saraiva MCP, Mestriner SF. Impact of oral and mental health over the quality of life of older people in a Family Health Unit. Rev Gaúch Odontol. 2020; 68: e20200043. doi: http://dx.doi.org/10.1590/1981-86372020000433676

18 - Martinelli KG, Belotti L, Poletto YM, Santos Neto ET, Oliveira, AE. Fatores associados ao cuidado de saúde bucal durante a gravidez. Arq Odontol. 2020; 56:1-9. doi: https://doi.org/10.7308/aodontol/2020.56.e16 
19 - Sakugawa KO, Martinelli KG, Esposti CDD, Pacheco Filho AC, Garbin CAS, Santos Neto ET. Saúde bucal e qualidade de vida de gestantes: a influência de fatores sociais e demográficos. Ciên Saúde Colet. 2020; 25: 2315-2324. doi: http://dx.doi.org/10.1590/1413-81232020256.24002018

20 - Zaze ACSF, Tondatti CA, Gamberini R, Pfau VJM. Relação de transmissibilidade da microbiota bucal entre pares mães-filhos. Arq Cienc Saúde UNIPAR, Umuarama. 2014; 8: 145-150. doi: https://doi. org/10.25110/arqsaude.v18i3.2014.5189

21 - Reis DM, Pitta DR, Ferreira HMB, Jesus MCP, Moraes MEL, Soares MG. Educação em saúde como estratégia de promoção de saúde bucal em gestantes. Ciênc Saúde Colet. 2010; 15(1): 269-276. doi: http://dx.doi. org/10.1590/S1413-81232010000100032

22 - Catao CDS, Gomes TA, Rodrigues RQF, Soares RCS. Evaluation of the knowledge of pregnant women about the relationship between oral diseases and pregnancy complications. Rev Odontol UNESP. 2015; 44(1): 59-65. doi: https://doi.org/10.1590/1807-2577.1078

23 - Rigo L, Dalazen J, Garbin RR. Impacto da orientação odontológica para mães durante a gestação em relação à saúde bucal dos filhos. Einstein. 2016; 14(2): 219-225. doi: http://dx.doi.org/10.1590/S167945082016AO3616

24 - Lemos LVFM, Myaki SI, Walter LRF, Zuanon ACC. Oral health promotion in early childhood: age of joining preventive program and behavioral aspects. Einstein. 2014; 12(1): 6-10. doi: http://dx.doi. org/10.1590/S1679-45082014AO2895

25 - Wrzosek T, Einarson A. Dental care during pregnancy. Can Fam Physician. 2009; 55(6): 598-9.

26 - Veiga Pessoa DM, de Macêdo GL. Autopercepção do pré-natal odontológico pelas gestantes de uma unidade básica de saúde. Revista Ciência Plural. 2018; 4(2): 60-72.

27 - Campos FAT, Carvalho AKFA, Cabral GMP, Silva CDAM, Ferreira MF, Tavares IPS. Manifestações bucais decorrentes da quimioterapia em crianças. Revista Campo do Saber. 2019; 4(5): 136-159.

28 - Leal AO, Rolim JIA, Muniz IAF, Farias IAP. Estudo dos parâmetros salivares de gestantes. Odontol Clín Cient. 2013; 12(1): 39-42.

29 - Cabral MCB, Santos TS, Moreira TP. Percepção das gestantes do Programa de Saúde da Família em relação à saúde bucal no município de Ribeirópolis, Sergipe, Brasil. Rev Port Sau Pub. 2013; 31(2):173-180. doi: http://dx.doi.org/10.1016/j.rpsp.2013.05.004

30 - Siqueira LQ, Baldicera CR, Daronco LS, Balsan LA. Possíveis prejuízos decorrentes do uso de tabaco e álcool durante a gestação. Rev Salusvita. 2017; 36(2): 587-99. 
31 - Martins LO, Pinheiro RPS, Arantes DC, Nascimento LS, Santos Júnior PB. Assistência odontológica à gestante: percepção do cirurgiãodentista. Rev Pan-Amaz Saude. 2013; 4(4): 11-18. doi: https://dx.doi. org/10.5123/S2176-62232013000400002

32 - Robles ACC, Grosseman S, Bosco VL. Práticas e significados de saúde bucal: um estudo qualitativo com mães de crianças atendidas na Universidade Federal de Santa Catarina. Ciênc. saúde colet. 2010; 15 (Suppl 2). doi: https://doi.org/10.1590/S1413-81232010000800033

33 - Silva RM, Brasil CCP, Bezerra IC, Queiroz FFSN. Mobile health technology for gestational care: evaluation of the Gestação app. Rev Bras Enferm. 2019; 72 (Suppl 3): 266-273. doi: https:/doi. org/10.1590/0034-7167-2018-0641

34 - Teixeira NM, Abreu PTR, Oliveira CAS, Zocratto KBF. Conhecimento materno em relação aos cuidados com a saúde bucal do bebê. Odontol Clín Cient. 2017; 16(4): 275-280.

35 - Alves FLC, Castro EM, Souza FKR, Lira MCPS, Rodrigues FLS, Pereira LP. Grupo de gestantes de alto-risco como estratégia de educação em saúde. Rev Gaúcha Enferm. 2019; 40:e20180023. doi: https://doi. org/10.1590/1983-1447.2019.20180023

36 - Pio DAM, Oliveira MM. Educação em saúde para atenção à gestante: paralelo de experiências entre Brasil e Portugal. Saude Soc. 2014; 23(1): 313-324. doi: https://doi.org/10.1590/S0104-12902014000100025 


\title{
Perceptions and attitudes of first pregnancy women in relation to maternal and child oral health care: a qualitative study
}

\begin{abstract}
Objective: To identify the beliefs of first pregnancy women who influence the search for dental assistance and the adoption of care related to their oral health and that of their children. Material and method: Descriptive study with qualitative methodology. Interviews were conducted with a semi-structured script, which were audio recorded and later transcribed, with 20 first pregnancy women registered in six family health units in a municipality in the state of São Paulo. The data were categorized using the Content Analysis technique and analyzed using the Health Belief Model. Results: Three categories were identified: Perceptions of risks and benefits in the first pregnancy, Perceived severity and barriers in the first pregnancy, Overcoming barriers and adoption of oral health care. Pregnant women believed in susceptibility to disease transmission during pregnancy, in the possibility of dental procedures and certain habits presenting risks to their health and influencing the baby's development. The main barriers pointed out were the fear of dental procedures and the refusal to assist pregnant women, while the family, social networks and health promotion groups were the main information media. Conclusions: Approaching the perceptions, beliefs and attitudes of primiparous women can increase knowledge about health care, reveal myths, habits and learning in this period, contribute to the planning of actions based on dialogue and strengthening bonds between team professionals and pregnant women, and promoting oral health care during prenatal care.
\end{abstract}

KEYWORDS: Oral Health; Pregnant Women; Prenatal Care; Perception; Attitude.

\section{Como citar este artigo}

Fumagalli IHT, Lago LPM, Mestriner SF, Bulgarelli AF; Mestriner Júnior W. Percepções e atitudes de primigestas em relação à atenção em saúde bucal materno-infantil: um estudo qualitativo. Rev Odontol Bras Central 2021; 30(89): 44-63. DOI: 10.36065/robrac.v30i89.1463 